\title{
Approach for the Synthesis of Potent Antimicrobials Containing Pyrazole, Pyrimidine and Morpholine Analogues
}

\author{
Nisheeth C. Desai ${ }^{a *}$, Bonny Y. Patel ${ }^{a}$, Bharti P. Dave ${ }^{b}$ \\ ${ }^{a}$ Division of Medicinal Chemistry, Department of Chemistry \\ (DST-FIST Sponsored \& UGC NON-SAP) \\ Mahatma Gandhi Campus, Maharaja Krishnakumarsinhji Bhavnagar University, \\ Bhavnagar-364002, India \\ ${ }^{b}$ Department of Life Sciences, Maharaja Krishnakumarsinhii Bhavnagar University, \\ Bhavnagar-364002, India \\ E-mail: dnisheeth@rediffmail.com \\ Tel. No: 0278-2439852
}

Keywords: Pyrazole, Pyrimidine, 1,3-diphenyl-1H-pyrazole-4-carbaldehyde, Antimicrobial evaluation, MIC.

\begin{abstract}
The present study is in the interest of some synthesized novel derivatives containing 4(1,3-diphenyl-1H-pyrazol-4-yl)- $N$-(morpholinomethyl)-6-arylpyrimidin-2-amines pooled with different bio-active heterocycles such as pyrazole, pyrimidine and morpholine derivatives. The structures of newly synthesized compounds were elucidated by IR, ${ }^{1} \mathrm{H}$ NMR, ${ }^{13} \mathrm{C}$ NMR and mass spectral data. The synthesized compounds were evaluated for their in vitro antimicrobial activity against different bacterial and fungal strains using Mueller-Hinton Broth dilution method. On the basis of SAR studies, it was observed that the presence of electron withdrawing groups remarkably enhanced the antimicrobial activity of synthesized compounds.
\end{abstract}

\section{Introduction}

Pyrazoles and several N-substituted pyrazoles are known to possess numerous chemical, biological and medicinal applications because of their adaptable biological activities such as antimicrobial [1], antileukemia [2], antidepressant [3], anticonvulsant, antipyretic, antineoplastic and antitubercular [4]. It is considered a typical model of pyrazole containing, diaryl-heterocyclic template that is known to selectively inhibit (cyclooxygenase enzyme) COX-2 [5]. Some of the example of pyrazole based drug are: Lonazolac (Non-steroidal anti-inflammatory drug), Mepiprazole (Minor tranquilizer), Rimonabant (Anorectic antiobesity drug), Ramifenazone (Analgesic, antipyretic and anti-inflammatory agent), Fipronil (Broad-spectrum insecticide) (Fig. 1). A literature survey revealed that a number of biologically active compounds have been synthesized using pyrazole3(4)-carbaldehydes and therefore in first step we have selected 1,3-diphenyl-1H-pyrazole-4carbaldehyde as a starting material.

The pyrimidine base is found in thymine, cytosine and uracil; which are the essential building blocks of nucleic acids and is also found in Vitamin $\mathrm{B}_{1}$. The literature indicated that compounds having pyrimidine nucleus possess broad range of biological activities, like 5-fluorouracil as anticancer, idoxuridine and trifluridine as antiviral, zidovudine and stavudine as anti-HIV, trimethoprim, sulphamethiazine and sulphadiazine as antibacterial, sulphadoxin as antimalarial. Pyrimidine derivatives have remarkable pharmaceutical importance because of their biological activity as anti-HIV [6], antitubercular, antimicrobial [7], antidiabetic [8] and antiinflammatory [9]. It has been found that several DHPM derivatives possess biological activity, which has led to their use as antiplasmodial [10] and anticancer [11]. Substituted aminopyrimidine nuclei is the key structure marketed drugs such as antiatherosclerotic aronixil, anti-histaminic thonzylamine, antianxielytic buspirone, antihypertensive minoxidil and prazosin, anti-psoriatic enazadrem, Common examples of pyrimidine based drugs are: Bacimethrin (Antibiotics), Viomycin (Antitubercular drugs), Alobarbital (Sedative/hypnotic), Retrovir (Antiviral and anti-HIV), etc (Fig. 1). 
Promising diverse pharmacological activities are shown by various N-functionalized morpholines. They are reported to exert a number of important physiological activities, such as antibacterial, antifungal [12], platelet aggregation inhibitors [13], antiemetic [14] and antidepressants [15], Phenmetrazine was used as an appetite suppressant. It was firstly replaced by its equivalent phendimetrazine which functions as a prodrug (Fig. 1). In accordance with our previous work [16-18] and medicinal significance of pyrazole, pyrimidine and morpholine motifs, we decided to combine all these moieties in to one single frame with hybrid approach as potential antimicrobial agents.

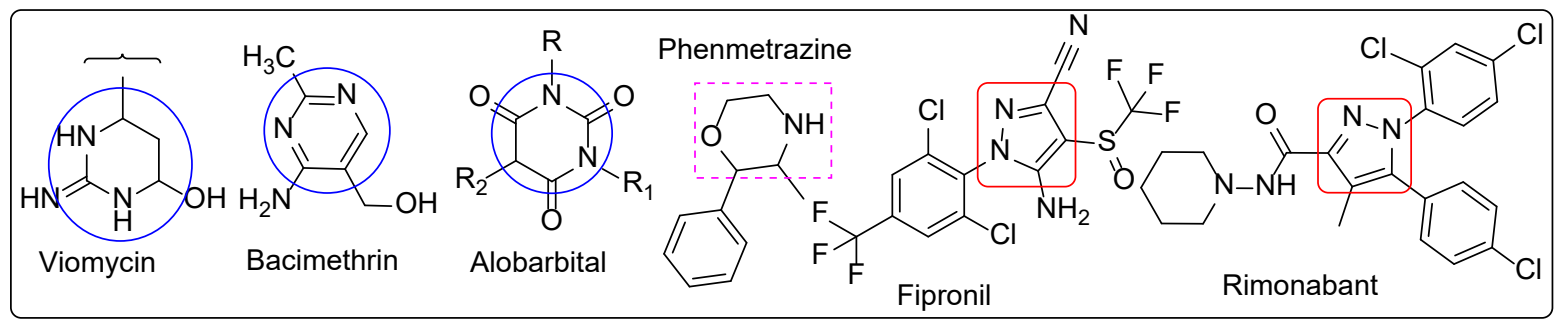

Fig. 1 Pyrazole, pyrimidine and morpholine-based commercially drugs

\section{Experimental}

\subsection{Materials and methods}

The required chemicals were purchased from E. Merck. Melting points were recorded on Gallenkamp apparatus and were left uncorrected. The completion of reaction and the purity of all compounds was checked on aluminum-coated TLC plates $60, \mathrm{~F}_{245}$ (E. Merck) using various solvent systems as mobile phase and visualized under ultraviolet (UV) light, or iodine vapor. Elemental analysis $(\% \mathrm{C}, \mathrm{H}, \mathrm{N})$ was carried out by a Perkin-Elmer $2400 \mathrm{CHN}$ analyzer. IR spectra were also recorded on Perkin Elmer FT-IR spectrophotometer. ${ }^{1} \mathrm{H}$ NMR and spectra were recorded on Varian Gemini $400 \mathrm{MHz}$ and ${ }^{13} \mathrm{C}$ NMR spectra on Varian Mercury-400, $100 \mathrm{MHz}$ in DMSO- $d_{6}$ as a solvent and tetramethylsilane (TMS) as an internal standard. Mass spectra were scanned on a Shimadzu LCMS 2010 spectrometer.

\section{Synthesis of 1,3-diphenyl-1H-pyrazole-4-carbaldehyde (1)}

1,3-diphenyl-1H-pyrazole-4-carbaldehyde (1) was prepared according to the literature method [19].

\section{Synthesis of 3-(1,3-diphenyl-1H-pyrazol-4-yl)-1-arylprop-2-en-1-ones (3a-o)}

3-(1,3-diphenyl-1H-pyrazol-4-yl)-1-arylprop-2-en-1-ones (3a-o) were prepared according to the literature method [20].

\section{Synthesis of 4-(1,3-diphenyl-1H-pyrazol-4-yl)-6-arylpyrimidin-2-amines (4a-o)}

4-(1,3-diphenyl-1H-pyrazol-4-yl)-6-arylpyrimidin-2-amines (4a-o) were prepared according to the literature method [21].

\section{General synthesis of 4-(1,3-diphenyl-1H-pyrazol-4-yl)- $N$-(morpholinomethyl)-6- arylpyrimidin-2-amines (5a-o)}

A mixture of compounds (4a-o) $(0.01 \mathrm{~mol})$, morpholine $(0.01 \mathrm{~mol})$ and formaldehyde $(0.02 \mathrm{~mol})$ was refluxed in ethanol for $10 \mathrm{~h}$. Excess of solvent was removed under reduced pressure and after cooling, the residue was poured onto crushed ice. Obtained solid product was filtered off, washed with water and crystallized from ethanol (95\%).

\section{4-(1,3-diphenyl-1H-pyrazol-4-yl)- $N$-(morpholinomethyl)-6-phenylpyrimidin-2-amine (5a)}

Yield: 70\%; mp $194^{\circ} \mathrm{C}$; IR ( $\left.\mathrm{cm}^{-1}\right)$ : 3311, 3236 (-N-H stretching), 3062 (-C-H aromatic stretching), 2956, 2914, 2852 (-C-H, $\mathrm{CH}_{2}$ stretching), 1597 (-C=N stretching), 1330 (-C-N stretching), 1222 (>C-O stretching), 1036 (-C-O-C stretching); ${ }^{1} \mathrm{H}$ NMR (DMSO- $\left.d_{6}\right) \delta$ ppm: $2.62\left(\mathrm{t}, 4 \mathrm{H}, \mathrm{N}\left(\mathrm{CH}_{2}\right)_{2}\right)$, $3.49\left(\mathrm{t}, 4 \mathrm{H}, \mathrm{O}\left(\mathrm{CH}_{2}\right)_{2}\right), 3.93\left(\mathrm{~s}, 2 \mathrm{H}, \mathrm{CH}_{2}\right), 6.75(\mathrm{~s}, 1 \mathrm{H}$, pyrimidine- $\mathrm{H}), 7.40-8.45(\mathrm{~m}, 15 \mathrm{H}, \mathrm{Ar}-\mathrm{H})$, 
8.32 (s, $1 \mathrm{H}, \mathrm{CH}$ of pyrazole), 10.20 (s, 1H, NH, $\mathrm{D}_{2} \mathrm{O}$ exch.); ${ }^{13} \mathrm{C}$ NMR (DMSO-d $\left.d_{6}\right) \delta$ ppm: 53.5, 66.4, 74.8, 101.2, 103.8, 119.8, 126.3, 127.6, 128.7, 129.3, 129.4, 131.6, 133.0, 135.6, 143.5, 145.8, 162.1, 162.3, 165.2; MS: $m / z=488.23$; Anal. Calcd. for $\mathrm{C}_{30} \mathrm{H}_{28} \mathrm{~N}_{6} \mathrm{O}: \mathrm{C}, 73.75 ; \mathrm{H}, 5.78$; N, 17.20; Found: C, 73.81; H, 5.74; N, $17.26 \%$.

\section{4-(1,3-diphenyl-1H-pyrazol-4-yl)- $N$-(morpholinomethyl)-6-(o-tolyl)pyrimidin-2-amine (5b)}

Yield: $68 \%$; mp $185^{\circ} \mathrm{C}$; IR $\left(\mathrm{cm}^{-1}\right)$ : 3313, 3229 (-N-H stretching), 3060 (-C-H aromatic stretching), 2962, 2919, 2850 (-C-H, $\mathrm{CH}_{2}$ stretching), $2930\left(\mathrm{C}-\mathrm{H}, \mathrm{CH}_{3}\right), 1610$ (-C=N stretching), 1321 (-C-N stretching), 1034 (-C-O-C stretching); ${ }^{1} \mathrm{H}$ NMR (DMSO-d $\left.d_{6}\right) \delta$ ppm: $2.46\left(\mathrm{~s}, 3 \mathrm{H}, \mathrm{CH}_{3}\right), 2.60(\mathrm{t}, 4 \mathrm{H}$, $\left.\mathrm{N}\left(\mathrm{CH}_{2}\right)_{2}\right), 3.52\left(\mathrm{t}, 4 \mathrm{H}, \mathrm{O}\left(\mathrm{CH}_{2}\right)_{2}\right), 3.94\left(\mathrm{~s}, 2 \mathrm{H}, \mathrm{CH}_{2}\right), 6.94(\mathrm{~s}, 1 \mathrm{H}$, pyrimidine- $\mathrm{H})$, 7.37-7.99 $(\mathrm{m}, 14 \mathrm{H}$, $\mathrm{Ar}-\mathrm{H}), 8.15$ (s, $1 \mathrm{H}, \mathrm{CH}$ of pyrazole), 10.19 (s, $1 \mathrm{H}, \mathrm{NH}, \mathrm{D}_{2} \mathrm{O}$ exch.); ${ }^{13} \mathrm{C}$ NMR (DMSO- $\left.d_{6}\right) \delta$ ppm: 18.7, 53.4, 66.3, 74.8, 101.2, 103.9, 119.9, 122.7, 126.2, 127.4, 128.6, 128.7, 129.2, 129.5, 129.7, $131.7,133.0,136.6,143.5,145.8,162.0,162.2,165.2 ; \mathrm{MS}: m / z=502.25$; Anal. Calcd. for $\mathrm{C}_{31} \mathrm{H}_{30} \mathrm{~N}_{6} \mathrm{O}: \mathrm{C}, 74.08 ; \mathrm{H}, 6.02 ; \mathrm{N}, 16.72$; Found: $\mathrm{C}, 73.96 ; \mathrm{H}, 6.06 ; \mathrm{N}, 16.62 \%$.

\section{4-(1,3-diphenyl-1H-pyrazol-4-yl)- $N$-(morpholinomethyl)-6-(p-tolyl)pyrimidin-2-amine (5c)}

Yield: $65 \%$; mp $180^{\circ} \mathrm{C}$; IR $\left(\mathrm{cm}^{-1}\right)$ : 3311, 3232 (-N-H stretching), 3061 (-C-H aromatic stretching), 2960, 2914, 2851 (-C-H, $\mathrm{CH}_{2}$ stretching), $2938\left(\mathrm{C}-\mathrm{H}, \mathrm{CH}_{3}\right), 1612(-\mathrm{C}=\mathrm{N}$ stretching), 1324 (-C-N stretching), 1031 (-C-O-C stretching); ${ }^{1} \mathrm{H}$ NMR (DMSO- $\left.d_{6}\right) \delta$ ppm: $2.36\left(\mathrm{~s}, 3 \mathrm{H}, \mathrm{CH}_{3}\right), 2.62(\mathrm{t}, 4 \mathrm{H}$, $\left.\mathrm{N}\left(\mathrm{CH}_{2}\right)_{2}\right), 3.50\left(\mathrm{t}, 4 \mathrm{H}, \mathrm{O}\left(\mathrm{CH}_{2}\right)_{2}\right), 3.92\left(\mathrm{~s}, 2 \mathrm{H}, \mathrm{CH}_{2}\right), 6.91(\mathrm{~s}, 1 \mathrm{H}$, pyrimidine- $\mathrm{H}), 7.35-7.96(\mathrm{~m}, 14 \mathrm{H}$, $\mathrm{Ar}-\mathrm{H}), 8.14$ (s, $1 \mathrm{H}, \mathrm{CH}$ of pyrazole), 10.16 (s, $1 \mathrm{H}, \mathrm{NH}, \mathrm{D}_{2} \mathrm{O}$ exch.) ${ }^{13} \mathrm{C}$ NMR (DMSO- $\left.d_{6}\right) \delta$ ppm: 21.7, 53.5, 66.5, 74.7, 101.3, 103.7, 119.9, 123.2, 126.2, 127.4, 128.6, 128.7, 129.5, 129.7, 131.5, $131.7,132.8,133.0,143.5,145.8,162.0,162.2,165.2 ; \mathrm{MS}: m / z=502.25$; Anal. Calcd. for $\mathrm{C}_{31} \mathrm{H}_{30} \mathrm{~N}_{6} \mathrm{O}$ : C, 74.08; H, 6.02; N, 16.72; Found: C, 74.00; H, 6.09; N, $16.76 \%$.

\section{4-(2-chlorophenyl)-6-(1,3-diphenyl-1 H-pyrazol-4-yl)- $N$-(morpholinomethyl)pyrimidin-2- amine (5d)}

Yield: $66 \%$; mp $174^{\circ} \mathrm{C}$; IR ( $\left.\mathrm{cm}^{-1}\right)$ : 3314, 3230 (-N-H stretching), 3058 (-C-H aromatic stretching), 2963, 2915, 2856 (-C-H, $\mathrm{CH}_{2}$ stretching), 1610 (-C=N stretching), 1322 (-C-N stretching), 1035 (-C-O-C stretching), 756 (C-Cl stretching); ${ }^{1} \mathrm{H}$ NMR (DMSO-d 6 ) $\delta$ ppm: $2.61\left(\mathrm{t}, 4 \mathrm{H}, \mathrm{N}\left(\mathrm{CH}_{2}\right)_{2}\right)$, $3.53\left(\mathrm{t}, 4 \mathrm{H}, \mathrm{O}\left(\mathrm{CH}_{2}\right)_{2}\right), 3.90\left(\mathrm{~s}, 2 \mathrm{H}, \mathrm{CH}_{2}\right), 6.89(\mathrm{~s}, 1 \mathrm{H}$, pyrimidine-H), 7.34-7.98 (m, 14H, Ar- $\mathrm{H})$, 8.12 (s, $1 \mathrm{H}, \mathrm{CH}$ of pyrazole), 10.17 (s, 1H, NH, $\mathrm{D}_{2} \mathrm{O}$ exch.); ${ }^{13} \mathrm{C}$ NMR (DMSO-d $) \delta$ ppm: 53.6, $66.3,75.0,101.3,103.8,119.9,126.2$, 127.4, 128.7, 128.9, 129.2, 129.7, 129.9, 130.1, 130.5, 131.5, $132.2,132.5,133.0,133.2,143.5,145.7,162.0,162.2,165.1 ;$ MS: $m / z=522.19$; Anal. Calcd. for $\mathrm{C}_{30} \mathrm{H}_{27} \mathrm{ClN}_{6} \mathrm{O}$ : C, 68.89; H, 5.20; N, 16.07; Found: C, 68.96; H, 5.26; N, $16.10 \%$.

\section{4-(4-chlorophenyl)-6-(1,3-diphenyl-1H-pyrazol-4-yl)- $N$-(morpholinomethyl)pyrimidin-2- amine (5e)}

Yield: $67 \%$; mp $168^{\circ} \mathrm{C}$; IR ( $\left.\mathrm{cm}^{-1}\right)$ : 3316, 3237 (-N-H stretching), 3062 (-C-H aromatic stretching), 2958, 2919, 2857 (-C-H, $\mathrm{CH}_{2}$ stretching), 1615 (-C=N stretching), 1320 (-C-N stretching), 1034 (-C-O-C stretching), 764 (C-Cl stretching); ${ }^{1} \mathrm{H}$ NMR (DMSO-d $) \delta$ ppm: $2.60\left(\mathrm{t}, 4 \mathrm{H}, \mathrm{N}\left(\mathrm{CH}_{2}\right)_{2}\right)$, $3.56\left(\mathrm{t}, 4 \mathrm{H}, \mathrm{O}\left(\mathrm{CH}_{2}\right)_{2}\right), 3.94\left(\mathrm{~s}, 2 \mathrm{H}, \mathrm{CH}_{2}\right), 6.88(\mathrm{~s}, 1 \mathrm{H}$, pyrimidine- $\mathrm{H}), 7.36-8.00(\mathrm{~m}, 14 \mathrm{H}, \mathrm{Ar}-\mathrm{H})$, $8.10\left(\mathrm{~s}, 1 \mathrm{H}, \mathrm{CH}\right.$ of pyrazole), $10.15\left(\mathrm{~s}, 1 \mathrm{H}, \mathrm{NH}, \mathrm{D}_{2} \mathrm{O}\right.$ exch.); ${ }^{13} \mathrm{C} \mathrm{NMR}$ (DMSO-d $) \delta$ ppm: 53.6, 66.3, 74.8, 101.3, 104.2, 119.7, 126.2, 127.6, 128.8, 128.9, 129.2, 129.5, 131.7, 133.0, 133.9, 134.3, 143.5, 145.9, 162.0, 162.2, 165.1; MS: $m / z=522.19$; Anal. Calcd. for $\mathrm{C}_{30} \mathrm{H}_{27} \mathrm{ClN}_{6} \mathrm{O}: \mathrm{C}, 68.89 ; \mathrm{H}$, 5.20; N, 16.07; Found: C, 68.83; H, 5.29; N, $16.12 \%$.

\section{4-(1,3-diphenyl-1H-pyrazol-4-yl)-6-(4-fluorophenyl)- $N$-(morpholinomethyl)pyrimidin-2-amine (5f)}

Yield: 72\%; mp $159^{\circ} \mathrm{C}$; IR ( $\left.\mathrm{cm}^{-1}\right)$ : 3320, 3234 (-N-H stretching), 3060 (-C-H aromatic stretching), 2955, 2922, 2854 (-C-H, CH stretching), 1616 (-C=N stretching), 1321 (-C-N stretching), 1118 (CF stretching), 1037 (-C-O-C stretching); ${ }^{1} \mathrm{H}$ NMR (DMSO- $\left.d_{6}\right) \delta$ ppm: 2.65 (t, 4H, N( $\left.\left.\mathrm{CH}_{2}\right)_{2}\right), 3.54$ (t, $\left.4 \mathrm{H}, \mathrm{O}\left(\mathrm{CH}_{2}\right)_{2}\right), 3.97\left(\mathrm{~s}, 2 \mathrm{H}, \mathrm{CH}_{2}\right), 6.87(\mathrm{~s}, 1 \mathrm{H}$, pyrimidine- $\mathrm{H}), 7.36-8.07(\mathrm{~m}, 14 \mathrm{H}, \mathrm{Ar}-\mathrm{H}), 8.12(\mathrm{~s}$, 
$1 \mathrm{H}, \mathrm{CH}$ of pyrazole), 10.16 (s, $1 \mathrm{H}, \mathrm{NH}, \mathrm{D}_{2} \mathrm{O}$ exch.); ${ }^{13} \mathrm{C}$ NMR (DMSO- $\left.d_{6}\right) \delta$ ppm: 53.6, 66.5, 74.9, $101.3,103.8,116.2,119.8,126.2$, 127.6, 128.8, 128.9, 129.2, 129.4, 130.6, 131.4, 131.7, 133.0, 134.3, 143.5, 145.9, 162.0, 162.2, 162.9, 165.1; MS: $m / z=506.22$; Anal. Calcd. for $\mathrm{C}_{30} \mathrm{H}_{27} \mathrm{FN}_{6} \mathrm{O}$ : C, 71.13; H, 5.37; N, 16.59; Found: C, 71.06; H, 5.29; N, $16.64 \%$.

\section{4-(4-bromophenyl)-6-(1,3-diphenyl-1 $H$-pyrazol-4-yl)- $N$-(morpholinomethyl)pyrimidin-2- amine (5g)}

Yield: $60 \%$; mp $147^{\circ} \mathrm{C}$; IR $\left(\mathrm{cm}^{-1}\right)$ : 3324, 3235 (-N-H stretching), 3061 (-C-H aromatic stretching), 2957, 2922, 2851 (-C-H, $\mathrm{CH}_{2}$ stretching), 1617 (-C=N stretching), 1324 (-C-N stretching), 1034 (-C-O-C stretching), 663 (-C-Br stretching); ${ }^{1} \mathrm{H}$ NMR (DMSO- $\left.d_{6}\right) \delta$ ppm: $2.62\left(\mathrm{t}, 4 \mathrm{H}, \mathrm{N}\left(\mathrm{CH}_{2}\right)_{2}\right)$, $3.57\left(\mathrm{t}, 4 \mathrm{H}, \mathrm{O}\left(\mathrm{CH}_{2}\right)_{2}\right), 3.94\left(\mathrm{~s}, 2 \mathrm{H}, \mathrm{CH}_{2}\right), 6.84(\mathrm{~s}, 1 \mathrm{H}$, pyrimidine- $\mathrm{H}), 7.41-8.03(\mathrm{~m}, 14 \mathrm{H}, \mathrm{Ar}-\mathrm{H})$, 8.12 (s, $1 \mathrm{H}, \mathrm{CH}$ of pyrazole), $10.16\left(\mathrm{~s}, 1 \mathrm{H}, \mathrm{NH}, \mathrm{D}_{2} \mathrm{O}\right.$ exch.); ${ }^{13} \mathrm{C} \mathrm{NMR}$ (DMSO-d $) \delta$ ppm: 53.6, 66.5, 74.7, 101.3, 103.8, 119.8, 123.2, 126.2, 127.6, 128.3, 128.8, 129.2, 129.4, 131.7, 132.1, 133.0, 134.8, 143.5, 145.9, 162.0, 162.2, 165.3; MS: $m / z=566.14$; Anal. Calcd. for $\mathrm{C}_{30} \mathrm{H}_{27} \mathrm{BrN}_{6} \mathrm{O}: \mathrm{C}$, 63.50; H, 4.80; N, 14.81; Found: C, 63.56; H, 4.87; N, $14.86 \%$.

\section{4-(1,3-diphenyl-1H-pyrazol-4-yl)-6-(2-methoxyphenyl)- $N$-(morpholinomethyl)pyrimidin-2- amine (5h)}

Yield: 68\%; mp $214^{\circ} \mathrm{C}$; IR $\left(\mathrm{cm}^{-1}\right)$ : 3325, 3239 (-N-H stretching), 3067 (-C-H aromatic stretching), 2951, 2924, 2857 (-C-H, $\mathrm{CH}_{2}$ stretching), 2840 (-OCH stretching), $1615(-\mathrm{C}=\mathrm{N}$ stretching), 1325 (-C-N stretching), 1032 (-C-O-C stretching); ${ }^{1} \mathrm{H}$ NMR (DMSO- $\left.d_{6}\right) \delta$ ppm: 2.63 (t, 4H, N(CH CH$_{2}$ ), $3.54\left(\mathrm{t}, 4 \mathrm{H}, \mathrm{O}\left(\mathrm{CH}_{2}\right)_{2}\right), 3.83\left(\mathrm{~s}, 3 \mathrm{H}, \mathrm{OCH}_{3}\right), 3.96\left(\mathrm{~s}, 2 \mathrm{H}, \mathrm{CH}_{2}\right), 6.82(\mathrm{~s}, 1 \mathrm{H}$, pyrimidine- $\mathrm{H}), 7.37-8.04$ $(\mathrm{m}, 14 \mathrm{H}, \mathrm{Ar}-\mathrm{H}), 8.14$ (s, $1 \mathrm{H}, \mathrm{CH}$ of pyrazole), 10.17 (s, 1H, NH, $\mathrm{D}_{2} \mathrm{O}$ exch.); ${ }^{3} \mathrm{C}$ NMR (DMSO- $\left.d_{6}\right)$ $\delta$ ppm: 53.6, 56.1, 66.7, 74.7, 101.3, 103.8, 111.5, 118.9, 119.7, 121.5, 126.2, 127.6, 128.3, 128.7, $129.2,129.4,129.7,131.1,131.7,132.1,133.0,143.4,145.9,157.4,162.0,162.2,165.3 ; \mathrm{MS}: \mathrm{m} / z=$ 518.24; Anal. Calcd. for $\mathrm{C}_{31} \mathrm{H}_{30} \mathrm{~N}_{6} \mathrm{O}_{2}$ : C, 71.79; H, 5.83; N, 16.20; Found: C, 71.82; H, 5.76; N, $16.28 \%$.

\section{4-(1,3-diphenyl-1H-pyrazol-4-yl)-6-(4-methoxyphenyl)- $N$-(morpholinomethyl)pyrimidin-2- amine (5i)}

Yield: 63\%; mp $205^{\circ} \mathrm{C}$; IR ( $\left.\mathrm{cm}^{-1}\right)$ : 3323, 3233 (-N-H stretching), 3064 (-C-H aromatic stretching), 2950, 2926, 2854 (-C-H, $\mathrm{CH}_{2}$ stretching), $2844\left(-\mathrm{OCH}_{3}\right.$ stretching), 1617 (-C=N stretching), 1327 (-C-N stretching), 1034 (-C-O-C stretching); ${ }^{1} \mathrm{H}$ NMR (DMSO-d $)_{6} \delta$ ppm: 2.62 (t, 4H, N( $\left.\left.\mathrm{CH}_{2}\right)_{2}\right)$, $3.52\left(\mathrm{t}, 4 \mathrm{H}, \mathrm{O}\left(\mathrm{CH}_{2}\right)_{2}\right), 3.88\left(\mathrm{~s}, 3 \mathrm{H}, \mathrm{OCH}_{3}\right), 3.97\left(\mathrm{~s}, 2 \mathrm{H}, \mathrm{CH}_{2}\right), 6.84(\mathrm{~s}, 1 \mathrm{H}$, pyrimidine- $\mathrm{H}), 7.34-8.07$ $(\mathrm{m}, 14 \mathrm{H}, \mathrm{Ar}-\mathrm{H}), 8.11$ (s, $1 \mathrm{H}, \mathrm{CH}$ of pyrazole), 10.12 (s, $1 \mathrm{H}, \mathrm{NH}, \mathrm{D}_{2} \mathrm{O}$ exch.); ${ }^{13} \mathrm{C}$ NMR (DMSO- $\left.d_{6}\right)$ $\delta$ ppm: 53.6, 55.8, 66.6, 74.7, 101.3, 103.8, 111.5, 118.9, 119.9, 121.5, 126.1, 127.6, 128.4, 128.7, 129.2, 129.3, 129.8, 131.0, 131.4, 132.1, 133.1, 143.5, 145.7, 160.6, 162.1, 162.3, 165.3; MS: $\mathrm{m} / \mathrm{z}=$ 518.24; Anal. Calcd. for $\mathrm{C}_{31} \mathrm{H}_{30} \mathrm{~N}_{6} \mathrm{O}_{2}$ : C, 71.79; H, 5.83; N, 16.20; Found: C, 71.83; H, 5.79; N, $16.26 \%$.

\section{2-(6-(1,3-diphenyl-1H-pyrazol-4-yl)-2-((morpholinomethyl)amino)pyrimidin-4-yl)phenol (5j)}

Yield: $61 \%$; mp $187^{\circ} \mathrm{C}$; IR ( $\left.\mathrm{cm}^{-1}\right)$ : 3408 (-OH stretching), 3326, 3239 (-N-H stretching), 3067 (-C$\mathrm{H}$ aromatic stretching), 2951, 2927, 2858 (-C-H, $\mathrm{CH}_{2}$ stretching), $1614(-\mathrm{C}=\mathrm{N}$ stretching), 1328 (-C-N stretching), 1036 (-C-O-C stretching); ${ }^{1} \mathrm{H}$ NMR (DMSO-d $d_{6} \delta$ ppm: $2.60\left(\mathrm{t}, 4 \mathrm{H}, \mathrm{N}\left(\mathrm{CH}_{2}\right)_{2}\right)$, $3.54\left(\mathrm{t}, 4 \mathrm{H}, \mathrm{O}\left(\mathrm{CH}_{2}\right)_{2}\right), 3.95\left(\mathrm{~s}, 2 \mathrm{H}, \mathrm{CH}_{2}\right), 6.87(\mathrm{~s}, 1 \mathrm{H}$, pyrimidine- $\mathrm{H}), 7.30-8.12(\mathrm{~m}, 14 \mathrm{H}, \mathrm{Ar}-\mathrm{H})$, $8.14\left(\mathrm{~s}, 1 \mathrm{H}, \mathrm{CH}\right.$ of pyrazole), $9.16(\mathrm{~s}, 1 \mathrm{H}, \mathrm{OH}), 10.17$ (s, 1H, NH, $\mathrm{D}_{2} \mathrm{O}$ exch.) ${ }^{13} \mathrm{C}$ NMR (DMSO$\left.d_{6}\right) \delta$ ppm: 53.6, 66.4, 74.9, 101.3, 103.9, 117.9, 119.9, 120.8, 121.8, 126.1, 127.6, 128.7, 129.2, 129.3, 131.1, 131.5, 131.6, 133.1, 143.5, 145.7, 155.7, 162.2, 162.3, 165.2; MS: $m / z=504.23$; Anal. Calcd. for $\mathrm{C}_{30} \mathrm{H}_{28} \mathrm{~N}_{6} \mathrm{O}_{2}$ : C, 71.41; H, 5.59; N, 16.66; Found: C, 71.49; H, 5.62; N, $16.60 \%$.

\section{3-(6-(1,3-diphenyl-1H-pyrazol-4-yl)-2-((morpholinomethyl)amino)pyrimidin-4-yl)phenol (5k)}

Yield: 59\%; mp $231^{\circ} \mathrm{C}$; IR ( $\left.\mathrm{cm}^{-1}\right)$ : 3411 (-OH stretching), 3324, 3235 (-N-H stretching), 3069 (-C$\mathrm{H}$ aromatic stretching), 2952, 2929, 2856 (-C-H, $\mathrm{CH}_{2}$ stretching), 1616 (-C=N stretching), 1326 
(-C-N stretching), 1037 (-C-O-C stretching); ${ }^{1} \mathrm{H}$ NMR (DMSO- $\left.d_{6}\right) \delta$ ppm: 2.63 (t, 4H, N( $\left.\mathrm{CH}_{2}\right)_{2}$ ), $3.57\left(\mathrm{t}, 4 \mathrm{H}, \mathrm{O}\left(\mathrm{CH}_{2}\right)_{2}\right), 3.91\left(\mathrm{~s}, 2 \mathrm{H}, \mathrm{CH}_{2}\right), 6.80(\mathrm{~s}, 1 \mathrm{H}$, pyrimidine- $\mathrm{H}), 7.28-8.02(\mathrm{~m}, 14 \mathrm{H}, \mathrm{Ar}-\mathrm{H})$, $8.16\left(\mathrm{~s}, 1 \mathrm{H}, \mathrm{CH}\right.$ of pyrazole), $9.29(\mathrm{~s}, 1 \mathrm{H}, \mathrm{OH}), 10.15\left(\mathrm{~s}, 1 \mathrm{H}, \mathrm{NH}, \mathrm{D}_{2} \mathrm{O}\right.$ exch.) ${ }^{13} \mathrm{C}$ NMR (DMSO$\left.d_{6}\right) \delta$ ppm: $53.6,66.4,74.7,101.4,103.9,115.9,119.9,120.1,126.2,127.6,128.7,129.2,129.3$, 130.5, 131.6, 133.1, 138.6, 143.5, 145.7, 155.7, 157.6, 162.2, 162.4, 165.2; MS: $\mathrm{m} / z=504.23$; Anal. Calcd. for $\mathrm{C}_{30} \mathrm{H}_{28} \mathrm{~N}_{6} \mathrm{O}_{2}$ : C, 71.41; H, 5.59; N, 16.66; Found: C, 71.48; H, 5.58; N, $16.64 \%$.

\section{4-(6-(1,3-diphenyl-1H-pyrazol-4-yl)-2-((morpholinomethyl)amino)pyrimidin-4-yl)phenol (5l)}

Yield: 73\%; mp $216^{\circ} \mathrm{C}$; IR ( $\left.\mathrm{cm}^{-1}\right): 3418$ (-OH stretching), 3321, 3232 (-N-H stretching), 3071 (-C$\mathrm{H}$ aromatic stretching), 2953, 2930, 2854 (-C-H, $\mathrm{CH}_{2}$ stretching), $1618(-\mathrm{C}=\mathrm{N}$ stretching), 1324 (-C-N stretching), 1036 (-C-O-C stretching); ${ }^{1} \mathrm{H}$ NMR (DMSO- $\left.d_{6}\right) \delta$ ppm: 2.65 (t, 4H, N( $\left.\left.\mathrm{CH}_{2}\right)_{2}\right)$, $3.57\left(\mathrm{t}, 4 \mathrm{H}, \mathrm{O}\left(\mathrm{CH}_{2}\right)_{2}\right), 3.92\left(\mathrm{~s}, 2 \mathrm{H}, \mathrm{CH}_{2}\right), 6.81(\mathrm{~s}, 1 \mathrm{H}$, pyrimidine- $\mathrm{H}), 7.24-8.07(\mathrm{~m}, 14 \mathrm{H}, \mathrm{Ar}-\mathrm{H})$, $8.13\left(\mathrm{~s}, 1 \mathrm{H}, \mathrm{CH}\right.$ of pyrazole), $9.34(\mathrm{~s}, 1 \mathrm{H}, \mathrm{OH}), 10.11\left(\mathrm{~s}, 1 \mathrm{H}, \mathrm{NH}, \mathrm{D}_{2} \mathrm{O}\right.$ exch.) ${ }^{13} \mathrm{C}$ NMR (DMSO$\left.d_{6}\right) \delta$ ppm: $53.6,66.6,74.7,101.4,103.9,116.5,119.8,120.1,126.2,127.6,128.4,128.7,128.9$, $129.2,129.3,131.6,132.8,143.5,145.7,158.6,162.2,162.4,165.2 ; \mathrm{MS}: \mathrm{m} / z=504.23$; Anal. Calcd. for $\mathrm{C}_{30} \mathrm{H}_{28} \mathrm{~N}_{6} \mathrm{O}_{2}$ : C, 71.41; H, 5.59; N, 16.66; Found: C, 71.45; H, 5.55; N, $16.62 \%$.

\section{4-(1,3-diphenyl-1 H-pyrazol-4-yl)- $N$-(morpholinomethyl)-6-(3-nitrophenyl)pyrimidin-2-amine} (5m)

Yield: 64\%; mp $241^{\circ} \mathrm{C}$; IR $\left(\mathrm{cm}^{-1}\right)$ : 3321, 3232 (-N-H stretching), 3071 (-C-H aromatic stretching), 2953, 2930, 2854 (-C-H, $\mathrm{CH}_{2}$ stretching), 1618 (-C=N stretching), 1485, 1351 (N-O asymmetric, symmetric stretching, $-\mathrm{NO}_{2}$ group), 1321 (-C-N stretching), 1039 (-C-O-C stretching); ${ }^{1} \mathrm{H}$ NMR (DMSO-d $\left.d_{6}\right) \delta$ ppm: $2.69\left(\mathrm{t}, 4 \mathrm{H}, \mathrm{N}\left(\mathrm{CH}_{2}\right)_{2}\right), 3.53\left(\mathrm{t}, 4 \mathrm{H}, \mathrm{O}\left(\mathrm{CH}_{2}\right)_{2}\right), 3.91\left(\mathrm{~s}, 2 \mathrm{H}, \mathrm{CH}_{2}\right), 6.85(\mathrm{~s}, 1 \mathrm{H}$, pyrimidine-H), 7.26-8.26 (m, $14 \mathrm{H}, \mathrm{Ar}-\mathrm{H}), 8.16(\mathrm{~s}, 1 \mathrm{H}, \mathrm{CH}$ of pyrazole $), 10.13\left(\mathrm{~s}, 1 \mathrm{H}, \mathrm{NH}, \mathrm{D}_{2} \mathrm{O}\right.$ exch.); ${ }^{13} \mathrm{C}$ NMR (DMSO- $\left.d_{6}\right) \delta$ ppm: 53.4, 66.4, 74.7, 101.4, 103.7, 119.8, 122.7, 123.9, 126.2, 127.6, 128.4, 128.9, 129.3, 129.5, 129.7, 131.6, 132.9, 143.5, 145.7, 158.6, 162.2, 162.4, 165.3; MS: $m / z=533.22$; Anal. Calcd. for $\mathrm{C}_{30} \mathrm{H}_{27} \mathrm{~N}_{7} \mathrm{O}_{3}: \mathrm{C}, 67.53 ; \mathrm{H}, 5.10 ; \mathrm{N}, 18.38$; Found: $\mathrm{C}, 67.50 ; \mathrm{H}$, $5.08 ; \mathrm{N}, 18.36 \%$.

\section{4-(1,3-diphenyl-1H-pyrazol-4-yl)- $N$-(morpholinomethyl)-6-(4-nitrophenyl)pyrimidin-2-amine} (5n)

Yield: $62 \%$; mp $262^{\circ} \mathrm{C}$; IR $\left(\mathrm{cm}^{-1}\right): 3321,3236$ (-N-H stretching), 3070 (-C-H aromatic stretching), 2950, 2936, 2857 (-C-H, $\mathrm{CH}_{2}$ stretching), 1621 (-C=N stretching), 1481, 1350 (N-O asymmetric, symmetric stretching, $-\mathrm{NO}_{2}$ group), 1326 (-C-N stretching), 1041 (-C-O-C stretching); ${ }^{1} \mathrm{H}$ NMR (DMSO-d $d_{6} \delta$ ppm: $2.70\left(\mathrm{t}, 4 \mathrm{H}, \mathrm{N}\left(\mathrm{CH}_{2}\right)_{2}\right), 3.51\left(\mathrm{t}, 4 \mathrm{H}, \mathrm{O}\left(\mathrm{CH}_{2}\right)_{2}\right), 3.93\left(\mathrm{~s}, 2 \mathrm{H}, \mathrm{CH}_{2}\right), 6.83(\mathrm{~s}, 1 \mathrm{H}$, pyrimidine-H), 7.24-8.31 (m, 14H, Ar-H), $8.21(\mathrm{~s}, 1 \mathrm{H}, \mathrm{CH}$ of pyrazole $), 10.17\left(\mathrm{~s}, 1 \mathrm{H}, \mathrm{NH}, \mathrm{D}_{2} \mathrm{O}\right.$ exch.); ${ }^{13} \mathrm{C}$ NMR (DMSO- $\left.d_{6}\right) \delta$ ppm: 53.4, 66.4, 74.9, 101.4, 103.8, 119.8, 124.4, 126.2, 127.6, $128.4,128.9,129.3,129.5,131.6,133.0,141.7,143.5,145.7,147.5,162.2,162.4,165.3 ; \mathrm{MS}: \mathrm{m} / z=$ 533.22; Anal. Calcd. for $\mathrm{C}_{30} \mathrm{H}_{27} \mathrm{~N}_{7} \mathrm{O}_{3}$ : C, 67.53; H, 5.10; N, 18.38; Found: C, 67.48; H, 5.12; N, $18.40 \%$.

\section{4-(2,4-dichlorophenyl)-6-(1,3-diphenyl-1H-pyrazol-4-yl)- $N$-(morpholinomethyl)pyrimidin-2- amine (5o)}

Yield: 69\%; mp $227^{\circ} \mathrm{C}$; IR ( $\left.\mathrm{cm}^{-1}\right)$ : 3314, 3233 (-N-H stretching), 3067 (-C-H aromatic stretching), 2958, 2914, 2861 (-C-H, $\mathrm{CH}_{2}$ stretching), 1615 (-C=N stretching), 1324 (-C-N stretching), 1034 (-C-O-C stretching), 739 (-C-Cl stretching); ${ }^{1} \mathrm{H}$ NMR (DMSO- $\left.d_{6}\right) \delta$ ppm: 2.61 (t, 4H, N( $\left.\left.\mathrm{CH}_{2}\right)_{2}\right)$, $3.58\left(\mathrm{t}, 4 \mathrm{H}, \mathrm{O}\left(\mathrm{CH}_{2}\right)_{2}\right), 3.96\left(\mathrm{~s}, 2 \mathrm{H}, \mathrm{CH}_{2}\right), 6.87(\mathrm{~s}, 1 \mathrm{H}$, pyrimidine- $\mathrm{H}), 7.30-8.08(\mathrm{~m}, 12 \mathrm{H}, \mathrm{Ar}-\mathrm{H})$, 8.10 (s, $1 \mathrm{H}, \mathrm{CH}$ of pyrazole), 10.18 (s, $1 \mathrm{H}, \mathrm{NH}, \mathrm{D}_{2} \mathrm{O}$ exch.); ${ }^{13} \mathrm{C}$ NMR (DMSO-d $d_{6} \delta$ ppm: 53.6, $66.3,74.8,101.3,104.2$, 119.7, 126.2 , 127.4, 128.0, 128.8, 128.9, 129.2, 129.5, 130.3, 130.9, 131.7, 133.0, 133.9, 135.3, 143.5, 145.9, 162.0, 162.2, 165.1; MS: $m / z=556.15$; Anal. Calcd. for $\mathrm{C}_{30} \mathrm{H}_{26} \mathrm{Cl}_{2} \mathrm{~N}_{6} \mathrm{O}: \mathrm{C}, 64.64 ; \mathrm{H}, 4.70 ; \mathrm{N}, 15.08$; Found: C, 64.69; H, 4.67; N, $15.01 \%$. 


\section{Results and discussion}

\subsection{Chemistry}

Synthetic strategies adopted to achieve the target compounds are depicted in Scheme $\mathbf{1}$. Here, scaffold $\mathbf{5}$ is a part of synthesis of new chemical entities in the form of antimicrobial agents. According to Scheme 1, the key chalcone derivatives 3-(1,3-diphenyl-1H-pyrazol-4-yl)-1-arylprop2-en-1-ones (3a-o) are used as precursors for the synthesis of title compounds (5a-o). Compounds (3a-o) were synthesized through the Claisen-Schmidt condensation of equimolar amounts of different acetophenone derivatives (2a-o) and compound 1,3-diphenyl-1 $H$-pyrazole-4-carbaldehyde (1) (Vilsmeier-Haack reaction adduct) by stirring the reactants in aqueous alcoholic solution containing potassium hydroxide at room temperature. To study the structure activity relationship with respect to antimicrobial properties, chalcone compounds (3a-0) on cyclocondensation reaction with guanidine hydrochloride in the presence of potassium hydroxide yielded compounds (4a-o). ${ }^{1} \mathrm{H}$ NMR spectrum of compound (4a) revealed a singlet characteristic peak for primary amine protons at $\delta=5.65 \mathrm{ppm}$ which was confirmed by disappearance of peak when exchange with $\mathrm{D}_{2} \mathrm{O}$. The IR spectra for (3a) showed the specific bands for the primary amine at 3344 and $3450 \mathrm{~cm}^{-1}$. After that, on Mannich reaction of 4-(1,3-diphenyl-1H-pyrazol-4-yl)-6-arylpyrimidin-2-amines (4a-o) with morpholine and formaldehyde in ethanol furnished 4-(1,3-diphenyl-1H-pyrazol-4-yl)- $N$ (morpholinomethyl)-6-arylpyrimidin-2-amines (5a-o). Structures of the isolated products (5a-0) were confirmed on the basis of their spectral data.<smiles>O=Cc1cn(-c2ccccc2)nc1-c1ccccc1</smiles>

(1)<smiles>[R]c1ccccc1C(C)=O</smiles>

(2a-o)<smiles></smiles>

(3a-o)

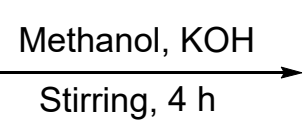

$\mathrm{NH}_{2} \mathrm{C}(=\mathrm{NH}) \mathrm{NH}_{2} \mathrm{HCl}$
Ethanol, $\mathrm{KOH}$ Reflux, $10 \mathrm{~h}$<smiles>[R]Cc1ccccc1-c1cc(-c2cn(-c3ccccc3)nc2-c2ccccc2)nc(NCN2CCOCC2)n1</smiles>

(5a-o)

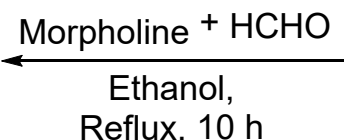

Reflux, $10 \mathrm{~h}$<smiles>[R]Cc1ccccc1-c1cc(-c2cn(-c3ccccc3)nc2-c2ccccc2)nc(N)n1</smiles>

$(4 a-0)$

Where $\mathbf{R}=-\mathrm{H},-2-\mathrm{CH}_{3},-4-\mathrm{CH}_{3},-2-\mathrm{Cl},-4-\mathrm{Cl},-4-\mathrm{F},-4-\mathrm{Br},-2-\mathrm{OCH}_{3},-4-\mathrm{OCH}_{3}$, $-2-\mathrm{OH},-3-\mathrm{OH},-4-\mathrm{OH},-3-\mathrm{NO}_{2},-4-\mathrm{NO}_{2},-2,4-(\mathrm{Cl})_{2}$

Scheme 1. Synthetic pathway for the synthesis of title compounds (5a-o)

IR spectrum of compounds (5a-o) revealed an absorption bands at 3325-3311, 3237-3229 and $1621-1597 \mathrm{~cm}^{-1}$ characteristic to secondary amine and cyanide group, respectively. Absorption bands at 2964-2950, 2936-2914 and 2861-2850 $\mathrm{cm}^{-1}$ were due to stretching vibration corresponding to methylene group. ${ }^{1} \mathrm{H}$ NMR spectrum revealed two triplet signals at $\delta=2.60-2.70$ and 3.49-3.57 ppm with coupling constants of nearly 4.5 and $4.8 \mathrm{~Hz}$ assignable to $\mathrm{N}\left(\mathrm{CH}_{2}\right)_{2}$ and $\mathrm{O}\left(\mathrm{CH}_{2}\right)_{2}$ of morpholine ring, respectively. Protons of methylene group showed as a singlet at $\delta=3.90-3.98$ ppm. The ${ }^{13} \mathrm{C}$ NMR spectrum of compounds (5a-o) revealed two important characteristic signals of carbons. One signals were displayed at $\delta=53.4-53.6$ and 66.3-66.7 ppm for $\mathrm{N}\left(\mathrm{CH}_{2}\right)_{2}$ and $\mathrm{O}\left(\mathrm{CH}_{2}\right)_{2}$, 
respectively, while methylene carbon were appeared downfield at $\delta=74.7-75.0 \mathrm{ppm}$. The mass spectrum revealed a molecular ion peaks were in agreement with proposed molecular weight and elemental analysis.

Compounds (5a-o) have pyrimidine core nucleus and are generated through cyclization, migration of $\alpha, \beta$ unsaturated double bonds, and diminishing of carbonyl group. A plausible mechanistic pathway for the formation of compounds (5a-o) is proposed in Scheme 2.
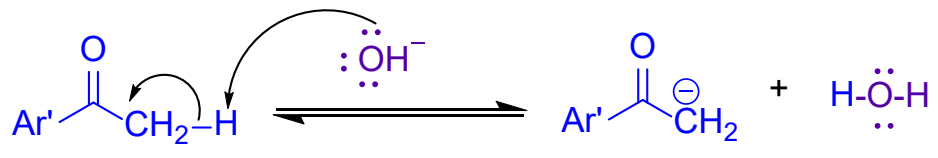

1

\section{2}

(2)

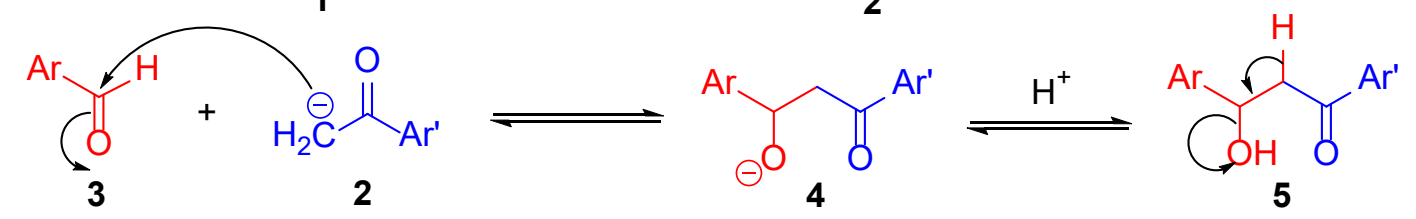

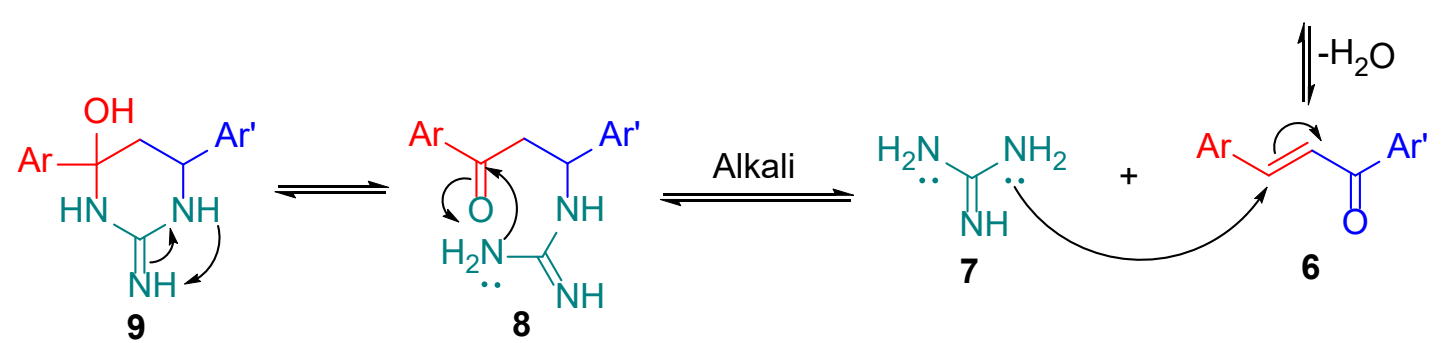<smiles>C=C1C(Br)=NC(N)=NC1CCCC</smiles>

10<smiles>Nc1nc(Br)cc(Br)n1</smiles>

11

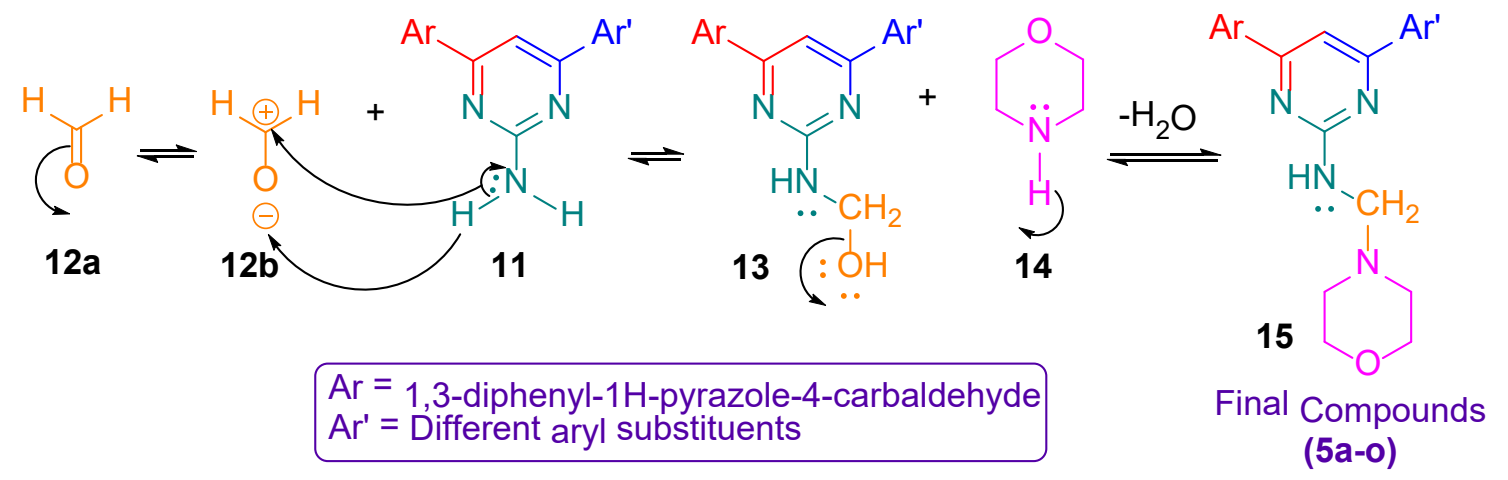

Scheme 2. Plausible mechanistic pathway of targeted compounds (5a-o)

\subsection{In vitro antimicrobial evaluation}

The activity of compounds was determined as per the National Committee for Clinical Laboratory Standards (NCCLS) protocol using Mueller-Hinton Broth (Becton-Dickinson, USA) [22-25]. All the newly synthesized compounds were evaluated against Gram-positive bacteria [Staphylococcus aureus (MTCC-96), Streptococcus pyogenes (MTCC-442)], Gram-negative bacteria Escherichia coli (MTCC-443), Pseudomonas aeruginosa (MTCC-1688)] and fungi [C. albicans (MTCC-227), A. niger (MTCC-282) and A. clavatus (MTCC-1323)] strains. 
Table 1 Antimicrobial screening of the compounds 5a-o

\begin{tabular}{|c|c|c|c|c|c|c|c|c|}
\hline \multirow{4}{*}{ Sr. No. } & \multirow{4}{*}{$-\mathbf{R}$} & \multicolumn{7}{|c|}{ MINIMUM INHIBITORY CONCENTRATIONS } \\
\hline & & \multicolumn{4}{|c|}{ FOR BACTERIA (MIC) in $\mu \mathrm{g} \mathrm{ml}^{-1}$} & \multirow{2}{*}{\multicolumn{3}{|c|}{ FOR FUNGI (MIC) in $\mu \mathrm{g} \mathrm{ml}^{-1}$}} \\
\hline & & \multicolumn{2}{|c|}{ Gram-negative } & \multicolumn{2}{|c|}{ Gram-positive } & & & \\
\hline & & E.c. & P.a. & S.a. & S.p. & C.a. & A.n. & A.c. \\
\hline 5a & $-\mathrm{H}$ & 500 & 250 & 500 & 500 & 1000 & 500 & 1000 \\
\hline $\mathbf{5 b}$ & $-2-\mathrm{CH}_{3}$ & 250 & 500 & $>1000$ & 250 & 1000 & 500 & 250 \\
\hline $5 c$ & $-4-\mathrm{CH}_{3}$ & 250 & 250 & 500 & $>1000$ & 1000 & 250 & 1000 \\
\hline 5d & $-2-\mathrm{Cl}$ & 250 & 250 & 250 & 500 & 250 & 100 & 50 \\
\hline $5 e$ & $-4-\mathrm{Cl}$ & 50 & 100 & 50 & 12.5 & $>1000$ & 250 & 250 \\
\hline $5 f$ & $-4-\mathrm{F}$ & 100 & 12.5 & 250 & 250 & 1000 & 250 & 250 \\
\hline $5 g$ & $-4-\mathrm{Br}$ & 250 & 250 & 500 & 250 & 250 & $\mathbf{5 0}$ & 100 \\
\hline $5 \mathrm{~h}$ & $-2-\mathrm{OCH}_{3}$ & 1000 & 250 & 500 & 500 & 1000 & 500 & 250 \\
\hline $5 \mathbf{i}$ & $-4-\mathrm{OCH}_{3}$ & 1000 & 500 & $>1000$ & 500 & $>1000$ & 500 & 250 \\
\hline $5 \mathbf{j}$ & $-2-\mathrm{OH}$ & 500 & 250 & 500 & 250 & $>1000$ & 250 & 1000 \\
\hline $5 \mathbf{k}$ & $-3-\mathrm{OH}$ & 250 & $>1000$ & 500 & 1000 & 1000 & 1000 & 500 \\
\hline 51 & $-4-\mathrm{OH}$ & 500 & 250 & $>1000$ & 250 & 1000 & 500 & 250 \\
\hline $5 \mathrm{~m}$ & $-3-\mathrm{NO}_{2}$ & 250 & 250 & 500 & 100 & 500 & 250 & 250 \\
\hline $5 n$ & $-4-\mathrm{NO}_{2}$ & 12.5 & 100 & 100 & 100 & 500 & 500 & 500 \\
\hline 50 & $-2,4-(\mathrm{Cl})_{2}$ & 100 & 250 & 25 & 250 & 1000 & 25 & $\mathbf{5 0}$ \\
\hline \multicolumn{2}{|c|}{ Ampicillin } & 100 & 100 & 250 & 100 & - & - & - \\
\hline \multicolumn{2}{|c|}{ Griseofulvin } & - & - & - & - & 500 & 100 & 100 \\
\hline
\end{tabular}

E.c.- Escherichia coli MTCC 443; P.a.- Pseudomonas aeruginosa MTCC 1688, S.a.- Staphylococcus aureus MTCC 96; S.p.- Staphylococcus pyogenes MTCC 442; C.a.- Candida albicans MTCC 227; A.n.- Aspergillus niger MTCC 282; A.c.- Aspergillus clavatus MTCC 1323

Amongst synthesized compounds (5a-o), many compounds exhibited antimicrobial potency that diverged from good to excellent. In preliminary screening, compounds 5a-o against the test microbes are listed in Table $\mathbf{1}$ along with MIC values of reference compounds ampicillin (for bacteria) and griseofulvin (for fungi). On the basis of antibacterial screening results from Table 1, compounds 5e, 5f, $\mathbf{5 n}$ and $\mathbf{5 0}$ were noticeably displayed outstanding antibacterial activity. For several pathogens, these compounds were more active than the reference drugs and the remaining compounds of the series possessed feeble antibacterial activity. Tabular data revealed that the presence of functional group at para position enhanced antibacterial activity as compared to ortho and meta substituted compounds.

We have discussed and compared antifungal activities based on standard drug Griseofulvin shown in Table 1. Compounds $\mathbf{5 m}\left(-3-\mathrm{NO}_{2}\right)$ and $\mathbf{5 n}\left(-4-\mathrm{NO}_{2}\right)$ were exhibited same MIC value as standard drug against $C$. albicans. Good activity possessed against $C$. albicans by compounds $\mathbf{5 d}$ (2-Cl) and $\mathbf{5 g}(-4-\mathrm{Br})$ at $\mathrm{MIC}=250 \mu \mathrm{g} \mathrm{ml}^{-1}$. Same compounds $\mathbf{5 d}$ and $\mathbf{5 g}$ also showed very good activity against $A$. niger, while highest inhibition $\left(25 \mu \mathrm{g} \mathrm{ml}^{-1}\right)$ exists in di-chloro substituted compounds, i.e. 5o $\left(-2,4-(\mathrm{Cl})_{2}\right)$. Moreover, compounds $5 \mathbf{d}$ and 50 having $-4-\mathrm{Cl},-2,4-(\mathrm{Cl})_{2}$ functional groups respectively, obsessed excellent activity against $A$. clavatus. Compounds having electron withdrawing groups in structure proves its significance to bacterial and fungal strains that makes a great imaginary impact in antimicrobial activity.

\subsection{Structure-activity relationship studies}

SAR studies revealed that the antimicrobial activity in heterocyclic class of bioactive molecules depends on the nature of the peripheral substituents and their spatial relationship within this skeleton. The presence of chloro, fluoro and nitro substituents at para position on substituted ring system had enriched the antibacterial activity of compounds compared to those of electron donating substituents. Compounds tend to lose its potency by changing the substitution pattern to ortho and meta position. Integration of electron donating groups such as methyl, methoxy and hydroxy 
reduced the antibacterial property. In rationality with the above results, electron withdrawing groups like fluoro and chloro on ortho and para substituted position showed optimal activity. It is determined from Table 1 that, a compound without any substitution does not display antimicrobial activity against a panel of all microorganisms.

\section{Conclusion}

We have synthesized newly compounds (5a-o) by conventional method with enlargement in yield of reactions. The synthesized compounds were screened for their in vitro antibacterial and antifungal activity against several bacterial and fungal strains. It is concluded from biological activity table that structural and electronic diversity of these products affects their biological activities. Compounds $\mathbf{5 d}, \mathbf{5 e}, \mathbf{5 f}, \mathbf{5 g}, \mathbf{5 n}$ and $\mathbf{5 0}$ are the most characteristic derivatives identified in present study because of their prominent in vitro antimicrobial potency. SAR studies reveal that when ortho and para positions are substituted by electron withdrawing group's fluoro, chloro, bromo and nitro; compounds revealed effective antimicrobial activity.

\section{Acknowledgements}

Authors are thankful to the University Grants Commission, New Delhi and Department of Science \& Technology, New Delhi for financial support under the NON-SAP and DST-FIST programs respectively.

\section{References}

[1] N.C. Desai et al., Synthesis and Evaluation of $N$-Substituted Thiazolidine-2,4-dione Containing Pyrazole as Potent Antimicrobial Agents, Anti-infact. Agents. 12 (2014) 85-94.

[2] L.C. Chou et al., Synthesis of furopyrazole analogs of 1-benzyl-3-(5-hydroxymethyl-2furyl)indazole (YC-1) as novel anti-leukemia agents, Bioorg. Med. Chem. 15 (2007) 1732-1740.

[3] M. Abdel-Aziz, G.E.A. Abuo-Rahma, A.A. Hassan, Synthesis of novel pyrazole derivatives and evaluation of their antidepressant and anticonvulsant activities, Eur. J. Med. Chem. 44 (2009) 34803487.

[4] D. Castagnolo et al., Synthesis, biological evaluation and SAR study of novel pyrazole analogues as inhibitors of Mycobacterium tuberculosis, Bioorg. Med. Chem. 16 (2008) 8587-8591.

[5] A. Palomer et al., Identification of novel cyclooxygenase-2 selective inhibitors using pharmacophore models, J. Med. Chem. 45 (2002) 1402-1411.

[6] N.C. Desai, G.M. Kotadiya, A.R. Trivedi, Studies on molecular properties prediction, antitubercular and antimicrobial activities of novel quinoline based pyrimidine motifs, Bioorg. Med. Chem. Lett. 24 (2014) 3126-3130.

[7] A. Barakat et al., Synthesis and structure investigation of novel pyrimidine-2,4,6-trione derivatives of highly potential biological activity as anti-diabetic agent, J. Mol. Str. 1098 (2015) 365-376.

[8] M.M. Hanna, New pyrimido[5,4-e]pyrrolo[1,2-c]pyrimidines: Synthesis, 2D-QSAR, antiinflammatory, analgesic and ulcerogenicity studies, Eur. J. Med. Chem. 55 (2012) 12-22.

[9] H. Kaur et al., Primaquine-pyrimidine hybrids: Synthesis and dual-stage antiplasmodial activity, Eur. J. Med. Chem. 101 (2015) 266-273.

[10] R.K. Yadlapalli et al., Synthesis and in vitro anticancer and antitubercular activity of diarylpyrazole ligated dihydropyrimidines possessing lipophilic carbamoyl group, Bioorg. Med. Chem. Lett. 22 (2012) 2708-2711.

[11] J.J. Bariwal ey al., Synthesis, characterization and anticancer activity of 3-aza-analogues of DP-7, Med. Chem. Res. 21 (2012) 4002-4009.

[12] R. Govindaraju, M. Gopalakrishnan, J. Thanusu, V. Kanagarajan, Synthesis, antibacterial and antifungal activities of biolabile (E)-1-4-morpholinophenyl)-3-aryl-prop-2-en-1-ones, Med. Chem. Res. 18 (2009) 341-350. 
[13] A. Ranise et al., 3,3-disubstituted 1-acyl-1-phenylthioureas with platelet antiaggregating and other activities, Farmaco. 46 (1991) 317-338.

[14] J.J. Hale et al., Phosphorylated Morpholine Acetal Human Neurokinin-1 Receptor Antagonists as Water-Soluble Prodrugs, J. Med. Chem. 43 (2000) 1234-1241.

[15] P. Avramova, N. Danchev, R. Buyukliev, T. Bogoslovova, Synthesis, Toxicological, and Pharmacological Assessment of Derivatives of 2-Aryl-4-(3-arylpropyl) morpholines, Arch. Pharm. 331 (1998) 342-346.

[16] N.C. Desai et al., Studies on Antimicrobial Evaluation of Some 1-((1-(1H-Benzo[d]imidazol-2yl)ethylidene)amino)-6-((arylidene)amino)-2-oxo-4-phenyl-1,2-dihydropyridine-3,5-dicarbonitriles, Synth. Commun. 45 (2015) 2701-2711.

[17] N.C. Desai et al., Synthesis, characterization and antimicrobial screening of thiazole based 1,3,4-oxadiazoles heterocycles, Res. Chem. Intermed. 42 (2015) 3039-3053.

[18] N.C. Desai et al., Synthesis, biological evaluation and molecular docking study of some novel indole and pyridine based 1,3,4-oxadiazole derivatives as potential antitubercular agents, Bioorg. Med. Chem. Lett. (2016).

[19] N.C. Desai et al., Facile synthesis of novel fluorine containing pyrazole based thiazole derivatives and evaluation of antimicrobial activity, J. Fluorine Chem. 142 (2012) 67-78.

[20] D.I. Brahmbhatt, A.R. Kaneria, A.K. Patel, N.H. Patel, Synthesis and antimicrobial screening of some 3-[4-(3-aryl-1-phenyl-1H-pyrazol-4-yl)-6-aryl-pyridin-2-yl] and 4-methyl-3-phenyl-6-[4(3-aryl-1-phenyl-1H-pyrazol4-yl)-6-aryl-pyridin-2-yl]coumarin, Ind. J. Chem. 49B (2010) 971-977.

[21] M.B. Siddesh, B. Padmashali, K.S. Thriveni, C. Sandeep, Synthesis of thiophene-linked pyrimidopyrimidines as pharmaceutical leads, J. Chem. Sci. 126 (2014) 821-826.

[22] National committee for clinical laboratory, Standards Methods for Dilution Antimicrobial Susceptibility Tests for Bacteria that Grow Aerobically approved standard, third ed. NCCLS Publication M7-A3, Villanova, PA, 1993.

[23] National committee for clinical laboratory standards, Reference Method for Broth Dilution Antifungal Testing of Yeasts, Proposed Standard, NCCLS Document M27-P; Villanova, PA, 1992.

[24] N.C. Desai, H.V. Vaghani, P.N. Shihora, A new hybrid approach and in vitro antimicrobial evaluation of novel 4(3H)-quinazolinones and thiazolidinone motifs, J. Fluorine Chem. 153 (2013) $39-47$.

[25] N.C. Desai, K.M. Rajpara, V.V. Joshi, Synthesis and characterization of some new quinoline based derivatives endowed with broad spectrum antimicrobial potency, Bioorg. Med. Chem. Lett. 22 (2012) 6871-6875. 